\title{
MEDIATE - a responsive environment designed for children with autism
}

\author{
Simone Gumtau, Paul Newland, Chris Creed, Simon Kunath \\ Centre for Responsive Environments, School of Art, Design \& Media, University of Portsmouth, UK \\ simone.gumtau@port.ac.uk, paul.newland@port.ac.uk,chris.creed@port.ac.uk, sck@unstablesound.net
}

\begin{abstract}
MEDIATE is a multi-sensory environment design for an interface between autistic and typical expression. It was designed as a space for creative expression and exploration via three sensory interfaces: visual, aural and tactile. The interaction with this digitally augmented world is under the child's control allowing them a sensory dialogue. It is as much a space for the user with autism to enjoy as an interface for relatives and carers to observe interactions and expressions.
\end{abstract}

MEDIATE is the outcome of a collaboration between Designers, Programmers and Psychologists from Spain, the Netherlands and the UK and was funded by the European Community. The expert psychology team on child development and autism informed the design process, which was essentially usercentred. The resulting outcome is successful for other user groups, but has benefited, in usability and innovation, from being constrained to a specific and challenging user group.

Multi-sensory, responsive, environment, autism, expression, digital, non-invasive, interface, psychology, interaction, pattern detection.

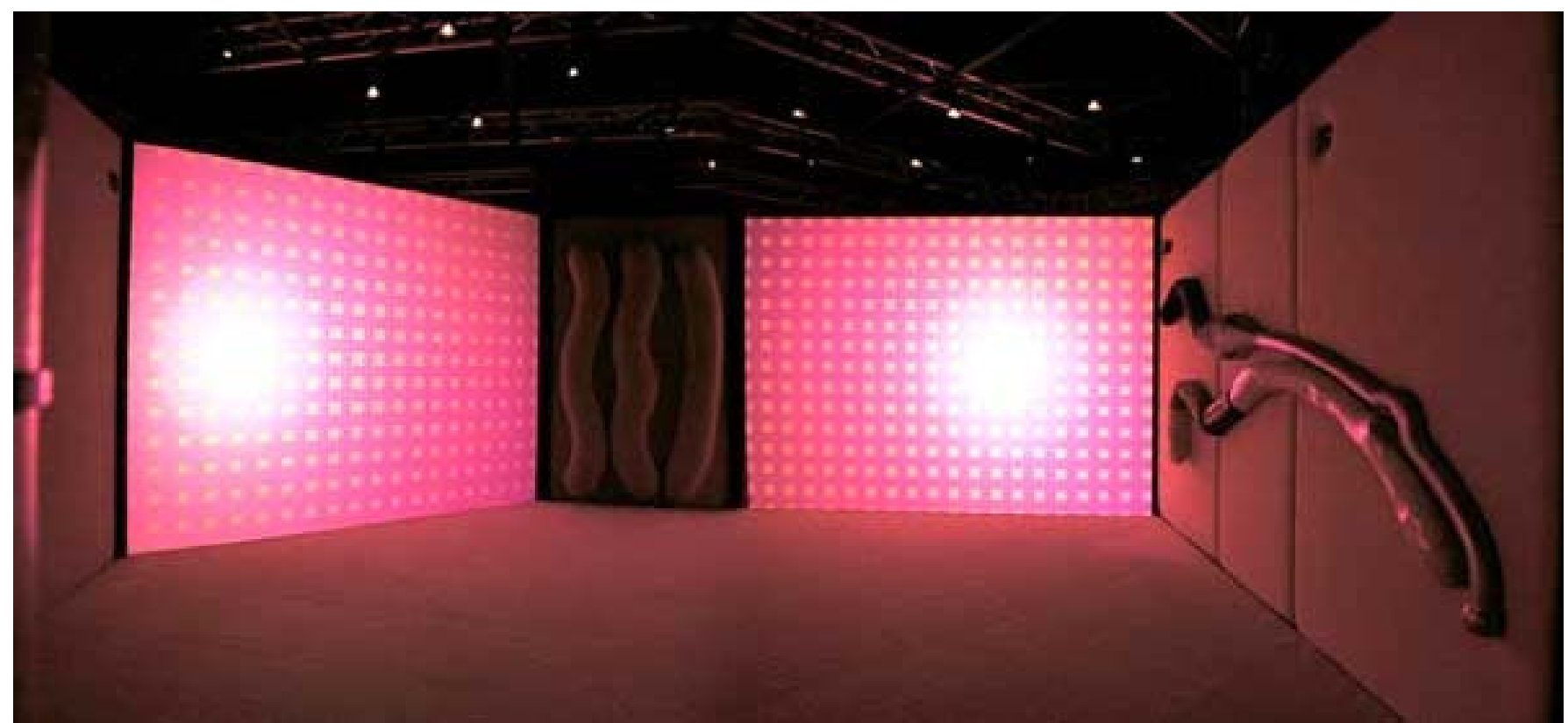

FIGURE 1: Panoramic view of the MEDIATE space

\section{MEDIATE OVERVIEW}

MEDIATE is a multi-sensory environment developed specifically for children with autism and limited or non-existent verbal skills. It is multi-sensory in the sense that it integrates visual, aural and touch via an input and output system. The environment is intended for one user at a time and the user creates feedback by movement, touch and making sounds. Carers or parents are not encouraged to facilitate or prompt interaction, although observation is recommended as part of the process to forge an understanding of the autism perspective.

The interaction consists of a dialogue between the child and the environment - when a child first enters the space, his/her body creates feedback that is directly related to the responses one would find in the physical world. This is to help the child find confidence in their explorations and to become aware that their actions and their bodies are having a direct effect. Once they have investigated a certain area, the feedback will become less direct and more abstract. This is to encourage creative exploration, while still maintaining a clear cause-and-effect relationship. 
Designing interactive systems for a challenging user group such as children with autism has focussed on developing therapeutic and/or educational applications (e.g. [1], [2], [3]). The psychologists on our team believe that our understanding of autism does not yet permit us to aim for such ambitious goals. This environment was not intentionally designed as a therapy for autism, although it was hoped that time spent being engaged in a sensory dialogue and experiencing a sense of agency is enjoyable and valuable time and might therefore offer benefits that could be called therapeutic in a sense. Fundamentally, MEDIATE is a space for the child with autism to be free from demands to fit into a neuro-typical world they usually find difficult to understand. Also, it allows us to observe and discover the way they shape their world through sensory interaction when engaged in this space.

In MEDIATE, sensory input can be controlled - the reducing or increasing of sensory input and the choice between different modalities is left entirely up to the user. It is designed as a space for children with autism to relax and have fun, discover a world in which they are in charge and find a method of releasing and expressing themselves through sensory channels [4], [5]. We had set a holistic design brief that proved strong enough to translate across all the developers involved, and the components integrated well into an inviting and satisfying responsive environment. It has the potential of being a research platform, a place of enjoyment and of developing its components for the sensory products market. The complex nature of the project meant that several lessons can be learned, including methodologies, design of specific artefacts and development of technology.

\section{ACESSIBLE DESIGN: PHYSICAL FEATURES AND INITIAL RESPONSES}

The environment itself is a space with six walls, two of which are back-projected screens. On entering, we immediately see the two screens come to life, detecting our presence by displaying a crude outline of our silhouette. We also notice the floor, which amplifies our footsteps with a crunchy sound feedback. To the right we find the "Tune Fork" mounted on the wall. This is a branched bas-relief structure covered in different textures. It is in the shape of a tune fork to motivate users to run their hands along it, to physically stretch themselves to reach the upper parts as well as providing smaller users access via the lower part. It reacts to our touch by sound feedback generated from microphones behind the textures. Between the two screens is the "Impression Wall" which consists of three cushioned shapes that allow the whole body to lean against and between. The shapes are foam-padded and provide vibratory feedback. Both "Tune Fork and "Impression Wall" are designed to be inviting to touch and exploration. Microphone coverage can detect audio input such as singing or clapping and the space reacts by echoing.

MEDIATE: illustration of view from the top

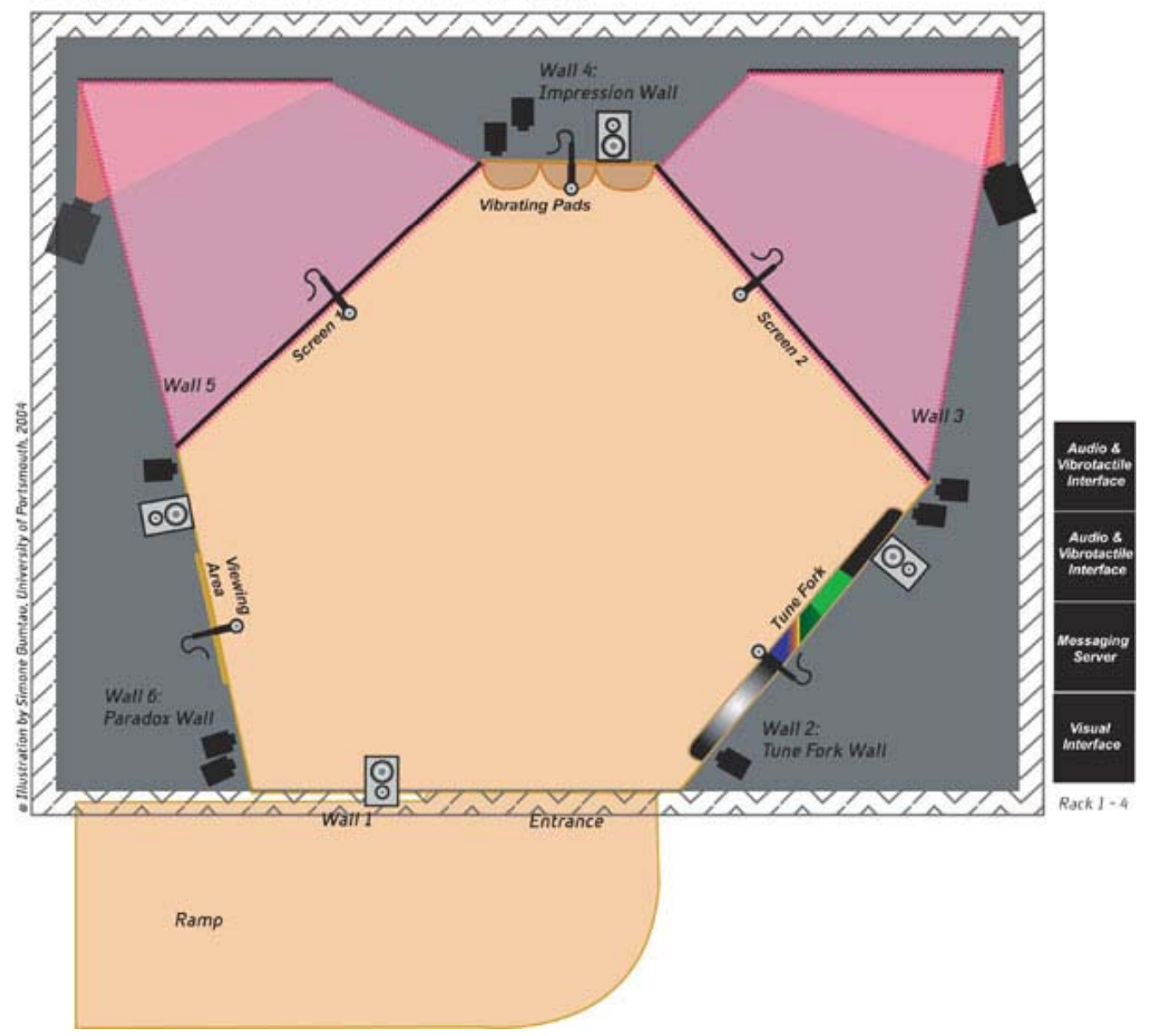

FIGURE 2: Diagram of the MEDIATE space 


\subsection{Technologies and Developments: Physical Space}

There are numerous technologies that were customized or developed for use in MEDIATE that can benefit the general issue of inclusive design. Even though autism is considered a social or developmental disorder, physical disabilities were also considered throughout. Wheelchair access was enabled by a ramp. MEDIATE accomplishes a natural language of interaction via gestures, making it possible for users to access the digital world without prior training or any particular literacy. This environment was also designed to be transportable in order to facilitate user studies in three different countries.

\subsubsection{Visual System}

As we could not predict the acceptance for a given individual of additional clothing, attached transmitters or handheld devices, and did not wish to exclude those adverse to them, our detection system - whilst responding to full-body interaction - had to be non-invasive.

Movement of limbs, posture and position makes the whole environment responsive, however this sensing is accomplished through the 'Eyes-Web' system developed for MEDIATE by the originators (A. Camurri) [6] and the Institut Universitari de l'Audiovisual, Universitat Pompeu Fabra partners, which demanded extraordinary infrared illumination. A network of lighting tracks was devised with predetermined, accurately located fixing points onto the outer roof frame. To obtain useful images at the size of the 3 metre wide interactive displays, high brightness projectors with rear projection screens were required. To maintain high contrast and colour purity, the rear projection techniques require a virtual blackout in the 'booth' - i.e. the area behind the screen. To keep within the imposed space, very wide-angle lenses and large mirrors were employed to attain the necessary geometry. The two screens are touch responsive. To obtain the desired interactive response, the Eyes-web system ingeniously used signals derived from infrared cameras. These were placed directly in line alongside the projection equipment. The proximity and actions of the participant are correlated with the visual effects by observing their shadow.

\subsubsection{Interactive Floor}

Providing access by spatial movement, the floor is an electronic system that provides information to the central system's 'brain', to determine the user's approximate position in space. It also provides a signal that is used to directly generate amplified footstep sounds or act as a trigger for other synthesised or sampled signals to reflect the nature of the participant's actions.

The floor is a completely modular construction - each section no bigger than one metre square. Each panel would be required to withstand the necessary load bearing, capable of carrying the necessary electronic interfaces, raised from the base floor in order to create a free space for cableways beneath, and all sections had to be capable of firmly locking into position to create an apparently continuous surface. It was also desirable that the floor should exhibit a slightly 'springy' step. Thus the considerations were both in respect of the physical construction itself and the need to accommodate the necessary electronic interfaces; whilst allowing direct body contact with the floor to provide response at a resolution where cause and effect were obvious over its entire surface.

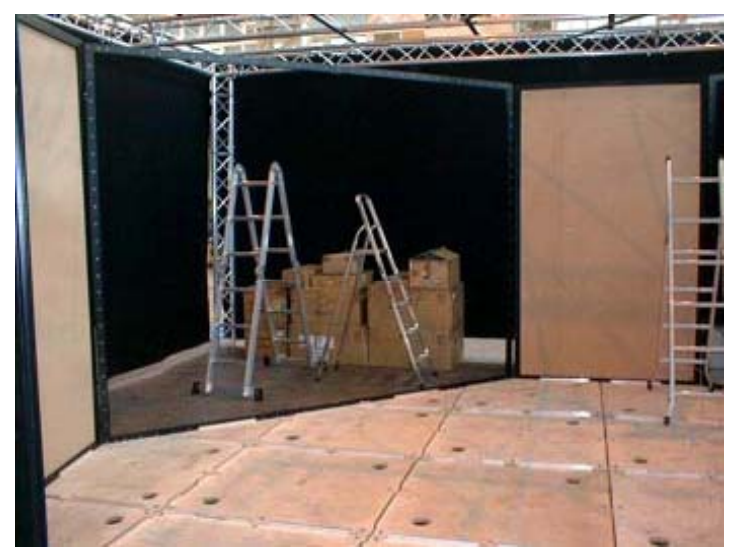

FIGURE 3: The interactive floor in construction

Each floor panel section was equipped with an electro-mechanical device to develop a 'tone' when compressed by a footstep which can be amplified directly to provide a sound similar to footsteps on a gravel path or, by means of an adjustable noise gate, provide midi information to key external devices. A special pre-amplifier capable of responding at sub-sonic frequencies was developed. Also, it was determined that these pre-amplifiers should be capable of deriving their power from a 48v 'phantom' source. This enabled each unit to be connected to the audio equipment via a single standard XLR plug and socket arrangement. A 'sandwich' construction method of marine ply, neoprene foam with an m.d.f. top plate was chosen for the floor. 


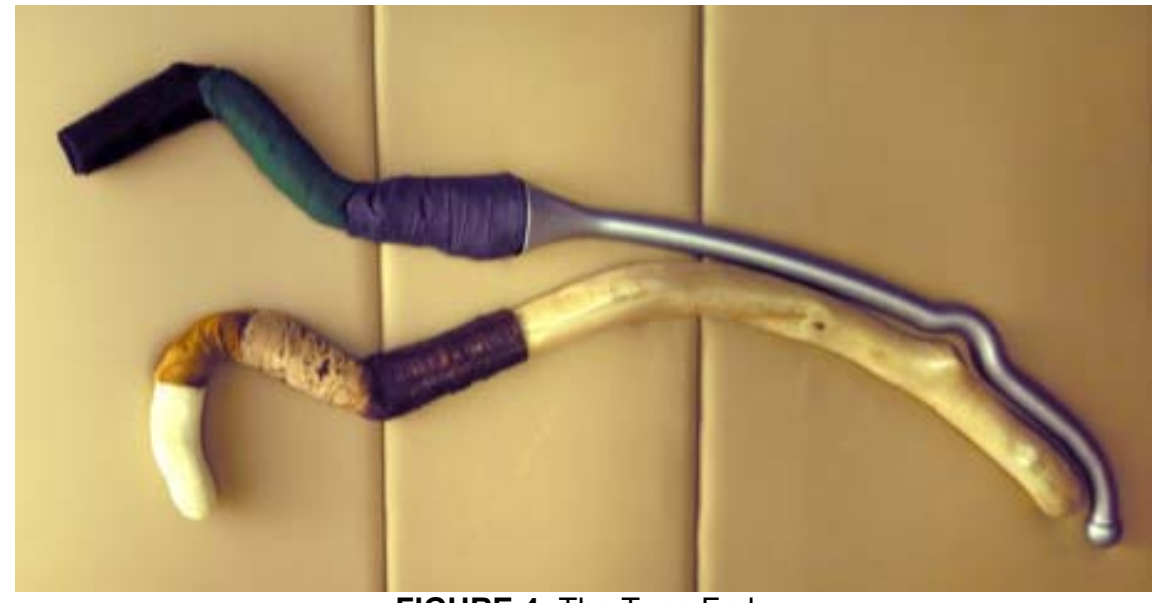

FIGURE 4: The Tune Fork

This is a new design to integrate interactive textures, which can be explored tactually and create musical responses. The interactive element has the same basic irregular shape as a cleft branch of a tree, but with the surface incorporating differing materials, thereby exhibiting various textures. The shape was designed to encourage movement and touch, i.e. running your hands along it and tapping. The materials join seamlessly and the whole item is interfaced electronically for touch response. A number of desirable textures (wood, bark, cork, fur, suede, metal, plastic, felt, studded rubber) were determined along the theme rough/smooth, incorporating organic and synthetic materials. A method of forming these to the required shape was developed. This involved forming individual moulds for each section and injecting polyurethane foam about a pre-shaped rigid 'back-bone'. Response to touch was achieved by employing small transducers imbedded into the foam close to the final surface. These were analogue devices and the signals obtained were to be directly amplified to produce an audible sound. In order to achieve sufficient 'gain', particularly at very low frequencies, a special pre-amplifier had to be developed. This device was similar to the transducers located in the floor panels (as described above). The basic analogue signals (pulses) derived from the 'Tune Fork' were also used to 'trigger' other electronic sounds and the computerised response management systems - all thereby increasing the potential range of reactions to the participant's initial touch. Again, as with the floor, but with variation and higher response resolution the guiding principle was gaining awareness of cause and effect in this area specifically with hand contact.

\subsubsection{Impression Wall}

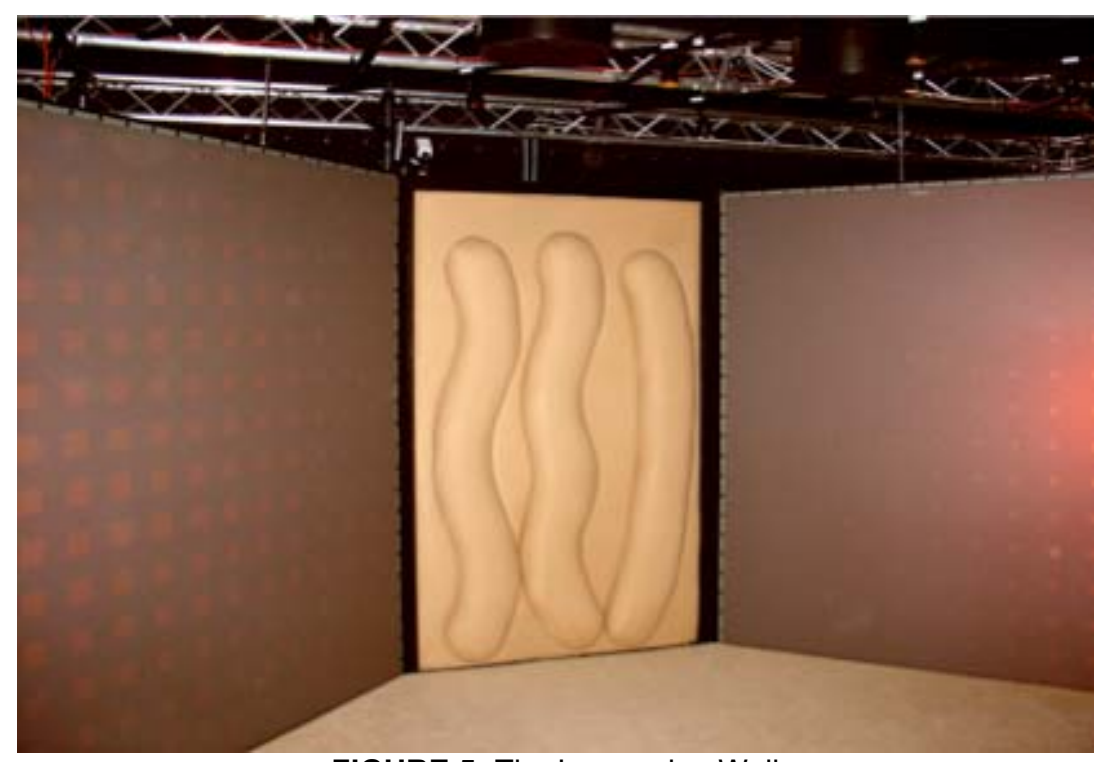

FIGURE 5: The Impression Wall

The Impression Wall provides vibro-tactile feedback by leaning against it. This feature offers a direct, proportional response to touch/pressure in the form of vibration of variable intensity (amplitude) and frequency. A series of vertical soft rounded snaking tubes were fitted with both sensors and vibrators. Due to the close proximity, a mechanical method for preventing feedback between the two elements was devised. Amplifiers specially biased for reproduction of low and sub-sonic frequencies were commissioned. The design arrangement was generated to enable semi-body envelopment, so that this area could subtly 'hug' the participant. 


\subsubsection{Sound System}

The sound system was devised so that 3 dimension "surround-sound" effects could be generated - the aural image could appear to be at any position horizontally and vertically. It was determined that this could be achieved economically by employing eight loudspeakers arranged with four at floor level and four at the maximum available height. An 8-channel amplifier provided the power for these loudspeakers. A loudspeaker was chosen that was affordable (particularly important when multiplied by a factor of 8 ), offered a reasonable response and, was of a style that could be rapidly flush mounted against the wall panels. In order to further assist with on-site construction, the standard connectors were removed and rugged XLR style plug / sockets were fitted. Here as before we were particularly keen to give sound localisable feedback to reinforce the immediacy and intimacy of cause and effect, the necessary precursor to evoke a sense of 'agency' for the participant.

\section{ACCESSIBLE DESIGN: USER DRIVEN DIALOGUE}

We started our design work with the knowledge that people with autism often display repetitive behaviour and by assuming that this repetition will increase when they are feeling uncomfortable or when they are disengaged from their immediate surroundings. We also assumed that being engaged with a situation, for this user group, usually meant it was enjoyable and we further assumed that continuing engagement was only likely if the user felt in control - experiencing a sense of 'agency'. It was therefore decided that the environment should discourage repetitive behaviour. We have to add here that we were designing for a user group that would not be able to tell us verbally what they liked and disliked: so called low function autism.

In order to keep coherence within the interaction, the system reacts to repetition by dimming down its feedback effects. Therefore the user does not associate repetitive behaviour with any particular effect, but rather with a process. At the design stage, there were concerns that an environment such as this could provide too much sensory stimulation, if crude amounts of activity led to greater effects. However the 'repetition = dimming' formula has the benefit that should the user be uncomfortable, he/she has the option of calming it.

However, if the user chooses to be novel in her/his behaviour, the environment's responses will become more complex, abstract and cross-modal.

The environment reacts to every user on the basis of their own particular set of idiosyncrasies and preferences. This is made possible by MEDIATE's real time pattern detection software "Signature Analyzer", which feeds data to a "Decision Maker", which determines the environment's response: a) 'evolution' - or b) 'dimming'.

This is the core essential to the project's approach. Bodily movements and sensor activation provide the input to "Signature", which primarily then creates a database of found behaviour patterns and their frequencies of occurrence. This in turn is used to alter the environment's state and the course of its evolution: found patterns are used to structure feedback; high levels of repetition lead to little change in the environment as opposed to low levels that are likely to allow environment change.

After an initial 'learning' period where patterns for this particular user, in this particular session are stored, "Signature" sends messages to "Decision Maker" indicating the levels of repetition/novelty being found.

An example relating to the floor can best indicate the environment's operation. As the child enters, his/her footsteps are amplified as a series of 'crunches' - like the sound made by walking through shingle on a beach, or treading on dry leaves. The user typically instantly recognises this sound effect as a resultant of their own walking. The 'crunches' continue whenever the child moves around. Meanwhile, "Signature" is collecting the user's behaviour patterns. After a while, the environment's "Decision Maker" asks whether there is a relatively high or low level of repetition at this time, as compared to this particular user's behaviour over the whole session so far. If repetition is low, then the "Decision Maker" will instruct the environment to change state. In the case of this example, footsteps will change from being a 'crunch' to being a 'pitched crunch'. The 'pitched crunches' now continue. Later, the "Decision Maker" asks itself again, based upon "Signature" data: "Is repetition low?". If the answer is again "yes", then further evolution is allowed - the 'crunch' drops out of the 'pitched crunch' leaving a 'musical' floor. Analogous changes occur with respect to other parts of the environment, including cross-modal effects, at later stages.

\section{METHODOLOGIES: DESIGN RESEARCH AND DEVELOPMENT}

\subsection{Expert Advice and Directing Aspects}

We were concerned to evolve a multi-sensory environment which was inclusive, but also investigate the potential to co-ordinate sensory modalities in a responsively augmented environment to enable types of expression beyond or different to those normally available in everyday life. Various approaches to gather such evidence have been attempted, one particularly fruitful way being the increasing accessibility of interactive media artists to technologies, which enlarge both the extent of interface engagements and the spectrum of media responsiveness. For example, enabling dancers' gesture and movement to orchestrate visual and aural accompaniments [9]. MEDIATE's approach as you have read is somewhat of a reversal to that strategy, we specifically chose a user group which were known to have particular difficulties in developing generative interactions in daily life. 
Our collaborative partner, the psychology team at the Institute of Psychiatry, at Kings College London and Goldsmiths College London, informed us through their knowledge of sensory processing with regards to autism, and direct clinical experience with our user group. They felt we should concentrate on the four principal, clinically recognised dysfunctional aspects which reduce the potential to bring forth novel interaction. Repetitive behaviour and a tendency for 'sticky' attention characterise the first aspect hence this necessitated that our environment, at some level, gain an awareness of over repeating cycles in the user's engagement.

A second area where impairment of generativity occurs is in the exhibition of pretend play, however this is usually judged in terms of ability to make believe social situations and relationships. Social settings are particularly problematic for children with autism, though it is not necessary to interpret this as a psychological deficit. One recent theory of autism [7] stands against deficit views of the condition, by suggesting that some aspects of autism represent a different psychological style. Here such individuals appear to show "weak central coherence", attending to parts rather than wholes, and processing information verbatim for detail rather than gist. Therefore, it is quite easy to understand how detailed processing may lead to overload in social settings where face, voice, action and so forth must be integrated and why such imaginative re-constructions are avoided by these individuals.

Against this pattern of impaired function, a number of research studies suggest hidden abilities and skills in autism. For example, savant skills are ten times more common in people with autism than in other disabled groups occurring in at least one in ten people. Autistic savants show striking abilities in the areas of art, music and number. Even those without known savant skills may have latent abilities that could be developed: Heaton's research has shown a high incidence of perfect pitch among children with autism who have no musical training [8]. She has also shown their ability to respond to the emotional aspects of music, despite the difficulties these children are typically thought to have in emotion processing. In the area of visual art, many show fine discrimination of details and ability to understand and express themselves through visual imagery. So here we concluded that lack of imagination should be countered by giving opportunity to control image, sound and vibration by the user's movements - a relationship that does not occur in this way within the neuro-typical world and thus a newness and novelty that can 'pull' new responses from the user and is not reliant on re-construction of social relationships.

The third aspect of concern, symbolic competence, is shown in recent research which has tried to summarise the autism phenomenon as 'mind blindness', but this label can also be applied to those who would consider themselves 'mind aware' when they are faced with autism. There is a breakdown in the ability to read each other's mind on both sides and the usual capacity for empathy is unavailable. Our users could be said to be 'locked into their own minds' largely because of others being 'mind blind' towards them. Consequently they often appear to be agitated and distressed. Among specialist teachers and researchers there is a general consensus and increasing realisation that it is appropriate to listen to individuals with autism and endeavour to give them 'voice'.

But how can you 'listen' to the completely non-social, non-speaking child? Lack of symbolic competence and this consequent conundrum is potentially circumnavigated we felt by allowing children with autism, through playfulness, interactivity and an integration of different sensory modalities, to create a sensory experience that is expressive of their own unique world. This framed our resolve to provide with MEDIATE a way for these individuals to build their own unique interactive world and show it to us so that we can more clearly understand the nature of their sensory world, thus opening up the possibility of dialogue in many non-verbal ways, facilitating a 'release' for them and an 'understanding' for us.

The fourth principal factor in curtailing generative interaction is the extent to which children with autism may have particular hypo- and / or hypersensitivity to sensory stimulus. For example, tolerance for a particular hue maybe markedly below average, whilst that for a specific band of sound frequencies maybe considerably above average. However, in a similar way to arguing the benefits of studying autism as a distinctive cognitive style rather than as a set of cognitive deficits, construing these individuals as having sensitivity differences rather than as suffering from sensory dysfunction is the more appropriate emphasis here. From a design perspective this meant responsiveness to individuals' particular hyper- and hyposensitivities, must be addressed by the capabilities of the 'signature' software. Although all four of these aspects could guide our design research we also understood the importance of individuals with first-hand experience of this condition being able to inform the on-going development.

\subsection{Participative User Study}

From the project's outset it was intended to involve people with autism and verbal skills, higher functioning than our target audience, to be informants during the design process. We were particularly fortunate to build a relationship of trust and an effective communication channel through a 10-year-old boy and his mother. Jonathan did not initially speak with us and in most circumstances would 'sign' with his mother, as this was a less ambiguous communication mode. Initially he acted as a consultant in the preliminary trials with vibro-tactile designs and then throughout the constructive and testing of the on-going full environment developments. With participation in early proto-type testing providing us with feedback, it was hoped our design iterations would then be useful even to people whose inner world we have no access to and who are therefore difficult to design for. The psychology team advised us to be careful with this method however as people with autism, as individuals, differ greatly in their idiosyncratic preferences and habits. One person might insist on including yellow squares at all times, while 
another might demand the same for green circles. There was the danger of particularising the environment to the proclivities of individuals rather than enabling a design accessible to all.

The design orientation was therefore more a general vision:

1) abstract - in a sense that it has no obvious connection to the social world and no figurative visual or aural representations, meaning no images or noises particularly referencing i.e. aeroplanes, windmills etc.

2) direct-physical - cause and effect in a sense that the design was led by an organic, natural theme and responses start off with being direct amplifications of the body. The body we thought was common and best known to every user regardless of their level on the autistic spectrum. The realisation of the body being the instigator of agency over the environment was also key to MEDIATE becoming literally accessible to everybody.

\section{PAN-EUROPEAN GROUP COLLABORATION}

One of the most challenging yet also most rewarding aspects of MEDIATE was undoubtedly the truly multidisciplinary approach enabled by the EU IST funding strategy. The University of Portsmouth, UK initiated, coordinated and integrated the project. They also had special responsibility for the "Decision Maker" software (the brain of the system) and the touch interface, including sensing surfaces and vibratory feedback. The Universitat Pompeu Fabra, Barcelona, Spain created the visual interface, looking after the visual tracking system as well as designing screen responses. The Hogeschool voor de Kunsten Utrecht, The Netherlands brought their music technology expertise to the project by developing the sound interface, designing all the aural responses in the environment. They are also responsible for the "Signature analyzer", the real time pattern detection software. Kings College London, UK in association with Goldsmiths College were our expert advisors on autism and user psychology from the start. They developed and saw through a pilot user study that was conducted in the three different countries MEDIATE has travelled to: UK, the Netherlands and Spain. The commercial partner responsible for the bespoke constructions that made MEDIATE a physical reality is Show Connections Ltd, Sussex, UK.

All partners in the MEDIATE project stressed that for them, the main benefit had been working with others from different disciplines, from different countries, and from the differences between a commercial and an academic outlook. They also agreed that the main and most important outcome arising from this heterogeneity of perspective was a product that they would not have come close to designing or realising on their own. Additionally, there was agreement that a huge benefit now would be the joint continuation and exploitation of MEDIATE both for academic research and for the building of new MEDIATE derived environments.

\section{CONCLUSION AND FUTURE WORK}

The prototype is completed and initial reports about user reactions have been collated and submitted to the funding body. After being installed and showcased at different locations throughout Europe attracting over 1,000 visitors, the environment is now situated in our research laboratory at the University of Portsmouth, UK.

With regard to our user group, there were three important objectives whose validity was assessed using experimental conditions - gaining a sense of agency; empathy and interpretation; promotion of creative activity. There were over 40 children across single sessions and we were fortunate enough to have nine children complete two sessions and so provide interpretable repeat data. From this we were able to conclude there was environment acceptance with children comfortable to (re)enter alone, that sensory modalities demonstrated agentful usage and some parents indicating higher than expected control from their children. Overall parental expectation altered, with greater enjoyment and less repetition than expected, whilst choice of modality focus was often unexpected, which together promoted greater likelihood of experiential exchange and parental insight. Confirmation of enhancement of creative activity was less easy to discern, though the environment's fostering features, repetitive fading and playful prompts were successful. An independent evaluation by the National Autistic Society was also favourable "from meeting with the parents and carers of children who had used MEDIATE it is clear to see that they felt it was a hugely beneficial experience that they would like to be able to continue to use."

With this view in mind a long-term study with young children with autism is currently on-going. Rewardingly too, the environment is attractive to a much larger audience than initially designed for. Designing a flexible system for a challenging and sometimes rigid user group has resulted in creating a rich sensory space independent from rules and demands of the social world. This seems to have a sometimes magic effect on people - not just children. We have indeed been approached by care institutions i.e. for people with profound learning difficulties, people with Downs syndrome or people with acute mental health problems. It was thought that a room like MEDIATE could be beneficial to their work. Even though a multi-sensory environment is especially of interest for people with disabilities whose access to sensory stimulation might be limited, the enjoyment and active engagement with it spreads to everyone.

We have all enjoyed creating MEDIATE and it was exhilarating to see the responses and hear the feedback. We are very pleased that the psychology research within MEDIATE is continuing and we are hoping to develop components of MEDIATE for the multi-sensory equipment market. 


\section{REFERENCES}

[1] Strickland, D. (1996) "A Virtual Reality Application with Autistic Children," PRESENCE: Teleoperators and Virtual Environments. MIT Press, vol 5.3. pp.319-329.

[2] Strickland, D. (1997, 1998) "Virtual Reality for the Treatment of Autism," Virtual Reality in Neuro-Psycho Physiology, G. Riva, ed., Amsterdam, Netherlands: los Press, pp. 81-86.

[3] Dautenhahn, K. (2000) )"Design issues on interactive environments for children with autism," Proc. $3^{\text {rd }}$ intl Conf. Disability, Virtual Reality \& Assoc. Tech., Alghero, Italy, pp. 153-159.

[4] MEDIATE official website, URL: http://www.port.ac.uk/mediate/ , last accessed June 2005

[5] Newland, P., Creed C., (2003) "MEDIATE: Steps Towards a Self-Organising Interface" Proc. Of the Fifth International CAiiA Research Conference on consciousness Reframed, R. Ascott, (Ed), Newport, Wales: University of Wales 2003

[6] Camurri, A., Coletta, P., Peri, M., Ricchetti, M., Ricci, A., Trocca, R., Volpe, G.,(2000) “A real-time platform for interactive performance," Proc. ICMC-2000, Berlin,.

[7] Happé, F.(1999) "Autism: Cognitive deficit or cognitive style?," Trends in Cognitive Sciences, vol. 3, pp. 216 222.

[8] Heaton, P., Hermelin, B., Pring, L., (1998)“Autism and pitch processing: a precursor for savant musical ability," Music Perception, vol. 15, pp. 291-305.

[9] Paine, G. in collaboration with Company in Space, "Incarnate," http://www.activatedspace.com (last visited March 2005). 